\title{
EFFECT OF EDDY CURRENTS IN A CORE CONSIST- ING OF CIRCULAR WIRES
}

\author{
By Chester Snow
}

ABSTRACT

This paper discusses mathematically the eddy currents in an infinitely long (or toroidal) core which consists of a bundle of wires of circular cross section. The wires are assumed to have a uniform and constant permeability and conductivity. The cases treated include those in which the rircuit inclosing the core may have resistance, inductance, and capacitance in series with the winding, and may also have an electromotive force which is any arbitrary function of the time. This is therefore more general than solutions heretofore published which cover only the case of a sinusoidal electromotive force.

The resulting formulas are applicable in problems connected with loading coils in communication circuits, with ignition apparatus, etc.

If a current $I(t)$ flows in a circuit with $N$ turns per $\mathrm{cm}$ in a long solenoid whose core consists of a bundle of $N^{\prime}$ long straight wires of circular section, the magnetic field $H$ is parallel to the wires and has the value of $4 \pi I(t)$ at all points in the air space, and hence over the surface of each wire. The field $H(r, t)$ at time $t$ at any point in a wire a distance $r$ from its center is then connected with the external current by the relation

$$
H(r t)=4 \pi N\left\{I(t)-\int_{-\infty}^{t} \dot{I}(\tau) d \tau \sum_{s=1}^{\infty} \frac{2 J_{\mathrm{o}}\left(\frac{r}{a} \alpha_{\mathrm{s}}\right)}{\alpha_{\mathrm{B}} J_{1}\left(\alpha_{\mathrm{B}}\right)} e^{\left.-\frac{\alpha_{\mathrm{s}}{ }^{\beta}(t-\tau)}{\beta}\right\}}\right.
$$

where $a$ is the radius of the core wire, $a_{\mathrm{B}}$ is the $s^{\text {th }}$ positive root of the Bessel's function $J_{0}(a)$ and $\beta$ is a characteristic of the core wire defined by $\beta=4 \pi \mu \lambda a^{2}$, where $\mu$ is its magnetic permeability and $\lambda$ is electrical conductivity. From this equation, by integration, the induced electromotive force by which the core reacts upon the current $I(t)$ may be computed, and this shows that the progress of the current $I(t)$ is determined by the integro-differential equation

$$
L \dot{I}(t)+R I(t)+\frac{Q(t)}{C}-4(1-\epsilon) L \beta \int_{0}^{\infty} I(t-\beta \tau) \sum_{s=1}^{\infty} \frac{e^{-\alpha_{s}{ }^{2} \tau}}{\alpha_{s}{ }^{2}} d \tau=V(t)
$$

where the applied emf is $V(t)$, the resistance of the circuit $R$, its series capacity $C$ with charge $Q(t)$. The total inductance of the circuit is $L$ and $\epsilon$ is a ratio defined by $\epsilon=1-\frac{4 \pi N^{2} \mu S_{\mu}}{L}$ where $S_{\mu}$ is the total cross-sectional area of core wires.

This shows that when the applied emf $V(t)$ is periodic with frequency $\frac{n}{2 \pi}$ the circuit acts as if its resistance were $R^{\prime}$ and inductance $L^{\prime}$ where $R^{\prime}$ and $L^{\prime}$ are functions of the frequency given by

$$
\frac{L^{\prime}-L}{L}=-(1-\epsilon) F_{1}(n \beta) \text { and } \frac{R^{\prime}-R}{n L}=(1-\epsilon) F_{2}(n \beta)
$$


where the functions $F_{1}$ and $F_{2}$ are expressed in terms of ber and bei functions of $\sqrt{n \beta}$ and are plotted against $\sqrt{n \beta}$ in the paper. When $V(t)$ is an arbitrary function of the time the current is found as an infinite series of exponential functions of the time, the first two terms of which may or may not be real. All the others are real exponentials corresponding to the time constants of the circuit, and all are determined by the roots of a certain transcendental equation which is discussed.

\section{CONTENTS}

I. Introduction Page

702

II. Magnetic field in the core wires in terms of the current._.

III. Case of periodic or alternating current

IV. Case of an arbitrary applied electromotive force... 712

V. Summary

\section{INTRODUCTION}

The variations in current in a coil whose core consists of a bundle of round iron wires causes a varying penetration of magnetic field into these wires, and this in turn reacts by electromagnetic induction upon the progress of the current, complicating it in such a manner that it is not usually amenable to mathematical treatment. However, the problem here treated is approximately applicable in certain cases. ${ }^{1}$

In this idealized case, to which the following treatment applies exactly, the magnetic permeability of the core is considered constant in the hope that an effective value may be assigned to this constant in the formulas obtained which may render them approximately correct. The demagnetizing effect of the ends of the coil is also neglected, so that the results are applicable only to closed magnetic circuits. In fact, the coil is treated as a very long straight solenoid. However, the results would obviously represent the behavior of a torus or anchor-ring type of coil if the diameter of the core is small compared to the mean radius of the ring.

\section{MAGNETIC FIELD IN THE CORE WIRES IN TERMS OF THE CURRENT}

If a circular cylinder of radius $a$, with its axis in the $z$ axis extending indefinitely in both directions, filled with a conductor whose conductivity is $\lambda$, and whose magnetic permeability is $\mu$, is subjected to a uniform and constant magnetic field parallel to its axis, the magnetic field intensity within it will be uniform and constant and the same as in the surrounding air. If, however, beginning at the time $t_{0}$, this field in the air is caused to change with time, due to external phenomena, in such a manner that it has the same value $H(t)$ at any time $t$, at all points on the boundary of the conducting cylinder, then

1 It was undertaken because of the necessity for some quantitative formula to account for the effect of eddy currents upon the voltage induced in a magneto. 
the circular eddy currents induced in the cylinder by this changing field will cause the value of the field within to lag behind the uniform value $H(t)$ which it would have in their absence. If we can find the manner in which the penetration of the magnetic induction into the circular core is influenced by these eddy currents, then it will be possible to take account of their reaction upon the external phenomena by which they are induced. The theory of the quasi-stationary state, which was first laid down by Maxwell, assumes that the time rate of change of the field vectors is slow relative to such rates as occur in optics, so that Ohm's law applies at every instant. This leads to the conclusion that within the conducting cylinder the field $H$ ( $z$ component only) must satisfy the partial differential equation

$$
4 \pi \lambda \mu H=\frac{1}{r} \frac{\partial}{\partial r}\left(r \frac{\partial H}{\partial r}\right)
$$

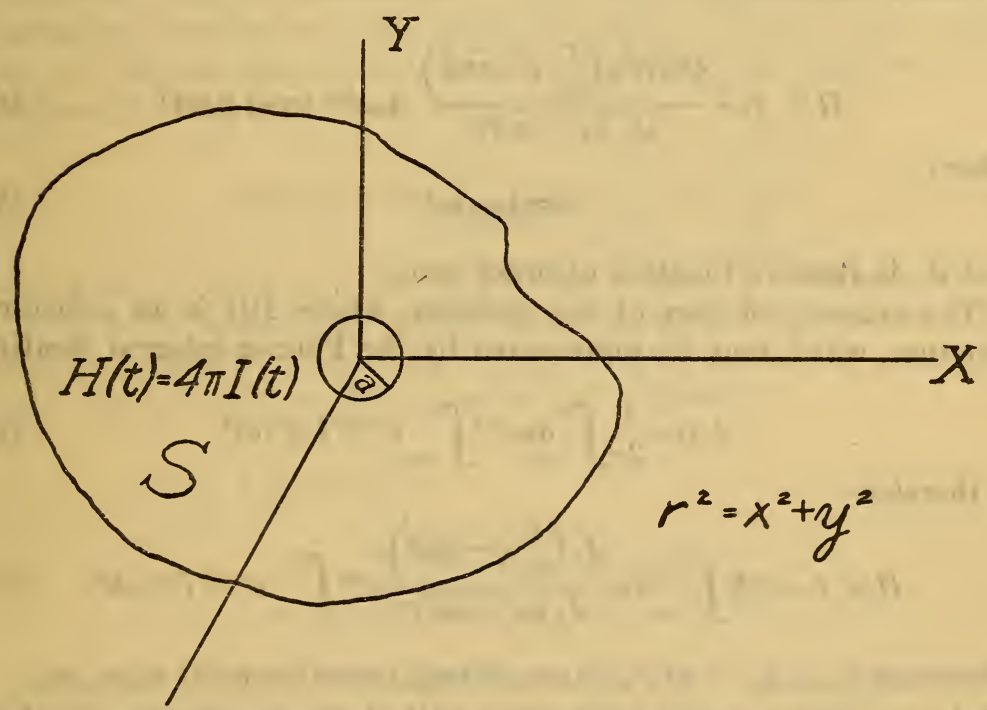

FIG. 1.-Section of infinite solenoid encircling one circular conducting cylinder

The second member of this equation is $\nabla^{2} H$, since $H$ is a function of $r$ only. This equation is derived by neglecting terms which correspond to the fact that the time in which field changes are propagated as waves from one part to another of the finite space is negligibly small compared to the time in which the induced currents spread through the medium. The equation (1) is therefore a diffusion equation rather than one of propagation with a definite velocity. The same assumption shows that in the surrounding air, or insulating medium, the field must rise or fall simultaneously with its rise or fall in the apparatus or neighboring circuits in which it originates. Thus (fig. 1) the plane section $S$ (of any finite shape) may 
be considered the boundary of the air, the section of an infinite cylinder inclosing the smaller circular conducting wire. There may be circular windings around this outer cylinder ( $N$ turns per $\mathrm{cm}$ ) so that the current circulating around unit length of the cylinder is $N I(t)$ at time $t$. At the inner boundary of $S$ (and at all points in the air within $S$ ) the field must, therefore, have the value $4 \pi N I(t)$. Consequently, the boundary condition, which holds at every instant $t$, at the boundary $r=a$ of the inner conducting cylinder is

$$
H(a, t)=4 \pi N I(t)
$$

The solution of (1) with the boundary condition (2) for the case where

$$
I(t)=I_{\mathrm{n}}(t)=A_{\mathrm{n}} e^{\mathrm{int}} \text { (real part) }
$$

is readily found to be

where

$$
H(r, t)=\frac{4 \pi N J_{\mathrm{o}}\left(\frac{r}{a} \sqrt{-i n \beta}\right)}{J_{\mathrm{o}}(\sqrt{-i n \beta})} A_{\mathrm{n}} e^{\mathrm{lnt}} \text { (real part) }
$$

$$
\beta \equiv 4 \pi \mu \lambda a^{2}
$$

and $J_{\mathrm{o}}$ is Bessel's function of order zero.

The general solution of the problem, where $I(t)$ is an arbitrary function, which may be represented by the Fourier integral identity

is therefore

$$
I(t)=\frac{1}{2 \pi} \int_{-\infty}^{\infty} d n e^{\operatorname{lnt}} \int_{-\infty}^{\infty} e^{-\mathrm{int} t^{\prime}} I\left(t^{\prime}\right) d t^{\prime}
$$

$$
H(r, t)=2 N \int_{-\infty}^{\infty} d n \frac{J_{\mathrm{o}}\left(\frac{r}{a} \sqrt{-i n \beta}\right)}{J_{\mathrm{o}}(\sqrt{-i n \beta})} e^{\mathrm{int}} \int_{-\infty}^{\infty} e^{-i \mathrm{nt} t^{\prime}} I\left(t^{\prime}\right) d t^{\prime}
$$

The roots $z_{1}, z_{2}, z_{3} \cdots$ of $J_{0}(z)$ are all real, hence the roots $n_{1}, n_{2}, n_{3} \cdots$ of $J_{0}(\sqrt{-i n \beta})$ are all on the upper half of the imaginary axis of $n$; that is, $n_{\mathrm{k}}=\frac{i z_{\mathrm{k}}{ }^{2}}{\beta}$. Consequently the path of the integration with respect to $n$ in the integral in equation (7) may be any path from $n=-\infty$ real to $n=+\infty$ real, which may be continuously deformed into the real axis without passing through any of the poles of the integrand which are the roots of $J_{0}(\sqrt{-i n \beta})=0$. For any application of physical interest, we may assume that $I(t)$ becomes identically zero when $t$ goes into the remote future or the remote past, thus insuring the convergence of the Fourier integral in equation (6). Under these circumstances, the order of integration in the double integral in equation (7) may be reversed, giving the solution for the magnetic field in the wire in the form 
where

$$
H(r, t)=4 \pi N \int_{-\infty}^{\infty} I\left(t^{\prime}\right) \psi\left(\frac{r}{a}, t-t^{\prime}\right) d t^{\prime}
$$

$$
\psi\left(\frac{r}{a}, t\right)=\frac{1}{2 \pi} \int_{-\infty}^{\infty} e^{\mathrm{int}} \frac{J_{0}\left(\frac{r}{a} \sqrt{-i n \beta}\right)}{J_{0}(\sqrt{-i n \beta})} d n
$$

The equation (8) appears to indicate that the field at time $t$ depends upon the values which the current shall have in the future, since the integration extends to all values greater than $t$. This is not the case, however, for it is found that the definition equation (9) makes $\psi\left(\frac{r}{a}, t\right)$ identically zero if $t$ is negative. This is shown by deforming the path of integration for the integral in equation (9) indefinitely downward in the complex $n$ plane, which is permissible since no poles of the integrand will be encountered. The integral then vanishes if $t$ is negative, because of the vanishing factor $e^{\operatorname{lnt}}=e^{-\mathbf{n}_{2} t} e^{\ln _{1} t}$ (where $n=n_{1}+i n_{2}$ ) for this vanishes when $n_{2}$ and $t$ are both negative. Consequently the equation ( 8 ) may be written

$$
H(r, t)=4 \pi N \int_{-\infty}^{t} I\left(t^{\prime}\right) \psi\left(\frac{r}{a}, t-t^{\prime}\right) d t^{\prime}
$$

For positive values of $t$ the path of the integral in equation (9) may be deformed indefinitely upward in the complex $n$ plane, thus reducing the integral to a series of contour integrals each taken around one and only one pole, so that equation (9) reduces to

$$
\begin{aligned}
\psi\left(\frac{r}{a}, t\right) & =0 \text { if } t<0 \\
& =-\frac{\partial}{\partial t} \sum_{s=1}^{\infty} \frac{2 J_{0}\left(\frac{r}{a} \alpha_{B}\right)}{\alpha_{B} J_{1}\left(\alpha_{B}\right)} e^{\frac{-\alpha_{s} s^{3} t}{\beta}} \text { if } t>0
\end{aligned}
$$

Using this expression in equation (10) gives ${ }^{2}$ after integration by parts and reduction by means of the formula

$$
\sum_{s=1}^{\infty} \frac{2 J_{0}\left(\frac{r}{a} a_{s}\right)}{a_{B} J_{1}\left(a_{B}\right)}=1 \text { if } \frac{r}{a}<1
$$

The solution equation (12) may also be derived by the method of Riemann for finding the temperature in the body when its surface temperature is a known function of the time. See Byerly: "Fourier's Series and Spherical, Cylindrical, and Ellipsoidal Harmonlcs," pp. 86-88. It is interesting to note that equation (12) also gives the course of the eddy currents created by a sudden change of magnetization. Thus if $I(t)=$ constant when $-\infty<t<0$ and $I(t)=0$ when $t>0$ then equation (12) gives

$$
H(r, t)=H_{0} 2 \sum_{\mathrm{S}=1}^{\infty} \frac{J_{0}\left(\frac{r}{a} \alpha_{s}\right)}{\alpha_{s} J_{1}\left(\alpha_{s}\right)} e^{\frac{-\alpha_{s}^{2} \mathrm{t}}{\beta}} \text { when } t>0
$$


where $a_{8}$ is the $s^{\text {th }}$ positive root of $J_{0}(a)=0$.

$$
\begin{aligned}
H(r, t) & =4 \pi N I(t)-4 \pi N \beta \int_{0}^{\infty} \dot{I}(t-\beta \tau) d \tau \sum_{\mathrm{s}=1}^{\infty} \frac{1}{2 J_{0}\left(\frac{r}{a} a_{\mathrm{B}}\right)} \frac{a_{\mathrm{B}} J_{1}\left(a_{\mathrm{B}}\right)}{e^{-\alpha_{\mathrm{s}}{ }^{2} \tau}} \\
& =4 \pi N I(t)-4 \pi N \int_{-\infty}^{t} \dot{I}\left(t^{\prime}\right) \sum_{\mathrm{s}=1}^{\infty} \frac{2 J_{0}\left(\frac{r}{a} a_{\mathrm{B}}\right)}{a_{\mathrm{B}} J_{1}\left(a_{\mathrm{g}}\right)} e^{\frac{-\alpha_{\mathrm{n}}{ }^{2}\left(t-t^{\prime}\right)}{\beta}} d t^{\prime}
\end{aligned}
$$

If the current $I(t)$ were a known function of the time, this equation would give the value of the axial component of magnetic field at a point in the core wire at a distance $r$ from its center at any time $t$. The current $I(t)$, however, will be influenced by the reaction of the field upon its circuit so that in general $I(t)$ will not be known.

It is evident from the fact that the field has the uniform value $4 \pi N I(t)$ at all points in the air within the section $S$, that there may be any number $N^{\prime}$ of equal core wires like the one just considered, in which the field will be given by the expression equation (12), where $r$ is the radial distance of a point from the axis of each wire no matter where it is situated within the section $S$. If $S_{\mathrm{a}}$ is the crosssectional area occupied by air and $S_{\mu}=N^{\prime} \pi a^{2}$, that occupied by the core material of permeability $\mu$ so that $S=S_{\mathrm{a}}+S_{\mu}$, then the magnetic flux through $S$ is by equation (12)

$$
\begin{aligned}
& \phi_{1}=\int \mathcal{S} \mu H d S=4 \pi N\left(S_{\mathrm{a}}+\mu S_{\mu}\right) I(t) \\
& -4 \pi N \mu S_{\mu} \beta \int_{0}^{\infty} \dot{I}(t-\beta \tau) d \tau \sum_{\mathrm{s}=1}^{\infty} \frac{4}{\alpha_{\mathrm{B}}^{2}} e^{-\alpha_{\mathrm{e}}^{2} \tau}
\end{aligned}
$$

Suppose the external circuit in which $I(t)$ flows has a resistance, $R$, and its terminals are connected to a capacity, $C$, and that it contains an applied electromotive force, $V(t)$, which is an arbitrary function of the time. If the current $I$ is considered positive when it encircles right-handedly the axis of the core, then the induced electromotive force in the circuit due to that part of the flux within $S$ is

$$
\begin{gathered}
V_{1}{ }^{\prime}=-N \frac{d \phi_{1}}{d t}=-4 \pi N^{2}\left(S_{\mathrm{a}}+\mu S_{\mu}\right) \dot{I}(t) \\
+4 \pi N^{2} \mu S_{\mu} \beta \int_{0}^{\infty} \ddot{I}(t-\beta \tau) d \tau \sum_{s=1}^{\infty} \frac{4}{a_{\mathrm{B}}{ }^{2}} e^{-\alpha_{\mathrm{s}}{ }^{2} \tau}
\end{gathered}
$$

The coefficient of $\dot{I}(t)$ on the right side of this equation is

$$
L_{\mathrm{o}}=4 \pi N^{2}\left(S_{\mathrm{a}}+\mu S_{\mu}\right)
$$


where $L_{0}$ is the self-inductance which the circuit would have if the core wires were nonconducting (that is, if $\beta=4 \pi \mu \lambda a^{2}=0$ ), and if the windings had no radial thickness. The actual inductance $L$ will be larger than $L_{0}$, due to the thickness of the windings. Moreover, it is possible to have additional inductance of any value $L_{1}$ inserted in this circuit which has no coupling with the field in $S$. Hence, if the inductance $L$ is arbitrary then the total induced electromotive force in the circuit is

$$
V^{\prime}=-L \dot{I}(t)+(1-\epsilon) L \beta \int_{0}^{\infty} \ddot{I}(t-\beta \tau) d \tau \sum_{s=1}^{\infty} \frac{4}{\alpha_{\beta}{ }^{2}} e^{-\alpha_{0}^{2} \tau}
$$

where

$$
L=L_{\mathrm{o}}+L_{1} \text { and } 1-\epsilon=\frac{4 \pi N^{2} \mu S_{\mu}}{L}
$$

Thus $\epsilon$ is a positive ratio less than 1 which might be small or exactly zero in limiting cases. The equation to determine the current is therefore

$$
L \dot{I}(t)+R I(t)+\frac{Q(t)}{C}-(1-\epsilon) L \beta \int_{0}^{\infty} \ddot{I}(t-\beta \tau) d \tau \sum_{s=1}^{\infty} \frac{4}{a_{s}^{2}} e^{-\alpha_{0}{ }^{2} \tau}=V(t)
$$

where $Q(t)$ is the charge on the condenser. Since $I(t)=\dot{Q}(t)$ this may be written

$$
L \ddot{Q}(t)+R \dot{Q}(t)+\frac{Q(t)}{C}-(1-\epsilon) L \beta \int_{0}^{\infty} \dddot{Q}(t-\beta \tau) d \tau \sum_{\mathrm{s}=1}^{\infty} \frac{4}{\sqrt{a_{\mathrm{s}}{ }^{2}}} e^{-\alpha_{\mathrm{s}^{2} \tau}}=V(t)
$$

Before attempting to solve the linear integro-differential equation (19) when the external electromotive force $V(t)$ is an arbitrary function of the time, we may first consider the special case where the latter is periodic.

\section{CASE OF PERIODIC ALTERNATING CURRENT}

\section{If}

then

$$
V(t)=V_{\mathrm{n}}(t)=B_{\mathrm{a}} e^{\mathrm{tnt}} \text { (real part) }
$$

$$
\begin{aligned}
Q(t) \equiv Q_{\mathrm{n}}(t) & =\frac{B_{\mathrm{n}} e^{\mathrm{Int}}}{L(i n)^{2}+\operatorname{Rin}+\frac{1}{C}-(1-\epsilon) L(i n)^{2} \sum_{\mathrm{s}=1}^{\infty} \frac{4 i n \beta}{a_{\mathrm{s}}{ }^{2}\left(a_{\mathrm{s}}{ }^{2}+i n \beta\right)}} \\
& =\frac{B_{\mathrm{n}} e^{\mathrm{Int}}}{(i n)^{2} L^{\prime}+i n R^{\prime}+\frac{1}{C}} \text { (real part) }
\end{aligned}
$$


where

$$
\begin{gathered}
\frac{L^{\prime}-L}{L} \equiv \frac{\triangle L}{L}=-(1-\epsilon) F_{1}(n \beta)=-(1-\epsilon) \sum_{\mathrm{s}=1}^{\infty} \frac{4 n^{2} \beta^{2}}{a_{\mathrm{s}}{ }^{2}\left(a_{\mathrm{s}}{ }^{4}+n^{2} \beta^{2}\right)} \\
\frac{R^{\prime}-R}{n L} \equiv \frac{\triangle R}{n L}=(1-\epsilon) F_{2}(n \beta)=(1-\epsilon) \sum_{\mathrm{s}=1}^{\infty} \frac{4 n \beta}{\frac{4}{a_{\mathrm{s}}{ }^{4}+n^{2} \beta^{2}}}
\end{gathered}
$$

There is no real value of $n$ for which the denominator in equation (21) vanishes. When $n$ is real, this constitutes the solution for the periodic case and $L^{\prime}$ and $R^{\prime}$ are the alternating current inductance and resistance of the circuit. The equations (22) and (23) show that the apparent resistance $R^{\prime}$ becomes infinite with infinite frequency, while the apparent inductance approaches a finite limit.

$$
L_{\infty}^{\prime}=L\left\{1-4(1-\epsilon) \sum_{\mathrm{s}=1}^{\infty} \frac{1}{a_{\mathrm{s}}^{2}}\right\}=\epsilon L
$$

The precise manner in which $L^{\prime}$ approaches this limit and $R^{\prime}$ becomes infinite will be found presently.

When the frequency, $f=\frac{n}{2 \pi}$, is so small that $n \beta=2 \pi \beta f<a_{1}{ }^{2}=(2.405)^{2}$, the sums in equations (22) and (23) may be expanded in ascending powers of $n \beta$ as follows:

$$
\begin{aligned}
& \sum_{\mathrm{s}=1}^{\infty} \frac{4 i n \beta}{\alpha_{\mathrm{s}}{ }^{2}\left(\alpha_{\mathrm{s}}{ }^{2}+i n \beta\right)}=4 \sum_{\mathrm{s}=1}^{\infty} \frac{i n \beta}{\alpha_{\mathrm{s}}{ }^{4}} \cdot \frac{1}{1+\frac{i n \beta}{\alpha_{\mathrm{s}}{ }^{2}}}=4 \sum_{\mathrm{s}=1}^{\infty} \frac{i n \beta}{\alpha_{\mathrm{s}}{ }^{4}} \sum_{\mathrm{k}=0}^{\infty}(-1)^{\mathrm{k}}\left(\frac{i n \beta}{\alpha_{\mathrm{s}}{ }^{2}}\right)^{\mathrm{k}} \\
& \quad=4 \sum_{\mathrm{k}=0}^{\infty}(-1)^{\mathrm{k}}(i n \beta)^{\mathrm{k}+1} \sum_{\mathrm{s}=1}^{\infty} \frac{1}{\alpha_{\mathrm{s}}{ }^{2 \mathrm{k}+4}}=4 \sum^{\infty}(-1)^{\mathrm{k}}(i n \beta)^{\mathrm{k}+1} S_{2 \mathrm{k}+4} \\
& \quad=4 n^{2} \beta^{2} \sum_{\mathrm{k}=0}^{\infty}(-1)^{\mathrm{k}}(n \beta)^{2 \mathrm{k}} S_{4 \mathrm{k}+6}+i 4 n \beta \sum_{\mathrm{k}=0}^{\infty}(-1)^{\mathrm{k}}(n \beta)^{2 \mathrm{k}} S_{4 \mathrm{k}+4}
\end{aligned}
$$

where

$$
S_{2 \mathrm{k}}=\sum_{\mathrm{s}=1}^{\infty} \frac{1}{\sqrt{\alpha_{\mathrm{g}} \mathrm{k}}}
$$


Hence the general expressions equations (22) and (23) may be replaced by the special ones

$$
\left.\begin{array}{l}
F_{1}(n \beta)=4(n \beta)^{2} \sum_{\mathrm{k}=0}^{\infty}(-1)^{\mathrm{k}}(n \beta)^{2 \mathrm{k}} S_{4 \mathrm{k}+6} \\
F_{2}(n \beta)=4 n \beta \sum_{\mathrm{k}=0}^{\infty}(-1)^{\mathrm{k}}(n \beta)^{2 \mathrm{k}} S_{4 \mathrm{k}+4}
\end{array}\right\} \begin{aligned}
& \text { if } \\
& n \beta<\alpha_{1}{ }^{2}=(2.405)^{2}
\end{aligned}
$$

The roots $a_{1}, \alpha_{2}, \alpha_{3} \ldots \ldots$ of $J_{0}(\alpha)=0$ as well as the sums of even powers of their reciprocals $S_{2 \mathbf{k}}$ are well known. In fact ${ }^{3}$

$$
S_{2}=2^{-2}, S_{4}=2^{-5}, S_{6}=\frac{1}{3} 2^{-8}, S_{8}=\frac{11}{3} 2^{-12}, S_{10}=\frac{19}{15} 2^{-13}
$$

and $S_{2 \mathrm{n}}$ may be computed by the relation

$$
\sum_{k=1}^{n} \frac{(-1)^{\mathrm{k}} 2^{2 \mathrm{k}} S_{2 \mathrm{k}}}{\underline{k-1} \underline{n-k}}=-\frac{1}{n \underline{n-1}}
$$

This may be derived from the expansion ${ }^{3}$

$$
\frac{z J_{1}(z)}{2 J_{0}^{-}(z)}=\sum_{n=1}^{\infty} z^{2 \mathrm{n}} S_{2 \mathrm{n}} \text { if }|z|<\alpha_{1}
$$

By means of equation (30) we may now show that the series occurring in equation (21), namely, $\sum_{\mathrm{s}=1}^{\infty} \frac{4 i n \beta}{\alpha_{\mathrm{s}}{ }^{2}\left(\alpha_{\mathrm{B}}{ }^{2}+i n \beta\right)}$ is identically $-\frac{J_{2}(\sqrt{-i n \beta)}}{J_{0}(\sqrt{-i n \beta})}$; that is, we may prove that

$$
\sum_{\mathrm{s}=1}^{\infty} \frac{4 z^{2}}{a_{\mathrm{s}}^{2}\left(a_{\mathrm{s}}{ }^{2}-z^{2}\right)}=\frac{J_{2}(z)}{J_{0}(z)}
$$

identically, that is for all values of $z$ for which each side has a meaning.

N. Nielsen: Handbuch der Theorie der Cylinderfunktionen, pp. 358-360. 
We shall first prove that equation (31) is true whenever the modulus of $z$ is less than $a$, the smallest positive root of $J_{0}(a)=0$. To do this we note that

$$
\begin{aligned}
\sum_{\mathrm{s}=1}^{\infty} \frac{4 z^{2}}{\alpha_{\mathrm{s}}{ }^{2}\left(\alpha_{\mathrm{s}}{ }^{2}-z^{2}\right)} & =-\sum_{\mathrm{s}=1}^{\infty} \frac{4}{\alpha_{\mathrm{s}}{ }^{2}}+\sum_{\mathrm{s}=1}^{\infty} \frac{4}{\alpha_{\mathrm{s}}{ }^{2}-z^{2}}=-1+4 \sum_{\mathrm{s}=1}^{\infty} \frac{1}{\sqrt{\alpha_{\mathrm{s}}{ }^{2}-z^{2}}} \\
& =-1+4 \sum_{\mathrm{s}=1}^{\infty} \frac{1}{\alpha_{\mathrm{s}}{ }^{2}} \sum_{\mathrm{n}=0}^{\infty}\left(\frac{z}{\alpha_{\mathrm{s}}}\right)^{2 \mathrm{n}}=-1+4 \sum_{\mathrm{n}=0}^{\infty} z^{2 \mathrm{n}} \sum_{\mathrm{s}=1}^{\infty} \frac{1}{\alpha_{\mathrm{s}}{ }^{2(\mathrm{n}+1)}} \\
& =-1+\frac{4}{z^{2}} \sum_{\mathrm{n}=0}^{\infty} z^{2(\mathrm{n}+1)} \sum_{\mathrm{s}=1}^{\infty} \frac{1}{\alpha_{\mathrm{s}}{ }^{2(\mathrm{n}+1)}}=-1+\frac{4}{z^{2}} \sum_{\mathrm{n}=1}^{\infty} z^{2 \mathrm{n}} \sum_{\mathrm{s}=1}^{\infty} \frac{1}{\alpha_{\mathrm{s}}{ }^{2 \mathrm{n}}} \\
& =-1+\frac{4}{z^{2}} \sum_{\mathrm{n}=1}^{\infty} S_{2 \mathrm{n}} z^{2 \mathrm{n}}=-1+\frac{4}{z^{2}}\left(\frac{z J_{1}(z)}{2 J_{\mathrm{o}}(z)}\right) \text { by }(30) \\
& =-1+\frac{2 J_{1}(z)}{z J_{0}(z)}=\frac{J_{2}(z)}{J_{0}(z)}
\end{aligned}
$$

This establishes equation (31) when $|z|<\alpha_{1}$. To show that equation (31) holds identically, that is for all points in the $z$ plane, let $g(z)$ denote the series on the left of equation (31), which evidently converges and defines a function of $z$ at all points in the $z$ plane except the points $z= \pm \alpha_{\mathrm{B}}, s=1,2,3 \ldots \ldots \infty \ldots$ which are all first-order poles for the function $g(z)$. It has no other singularities. But these are all first-order poles of the second member of equation (31), and there are no others. Consequently, the product $G(z)=g(z) J_{0}(z)$ is a function which is equal to $J_{2}(z)$ at all points $z$ inside the circle of radius $\alpha_{1}$. Furthermore, $G(z)$ and $J_{2}(z)$ have no poles in the finite part of the $z$ plane and must be identical everywhere, so that equation (31) is an identity. If we place $z^{2}=-i n \beta$ in equation (31) it gives the relation above stated, namely,

$$
\sum_{\mathrm{s}=1}^{\infty} \frac{4 i n \beta}{a_{\mathrm{s}}{ }^{2}\left(a_{\mathrm{s}}{ }^{2}+i n \beta\right)}=-\frac{J_{2}(\sqrt{-i n \beta)}}{J_{\mathrm{o}}(\sqrt{-i n \beta)}}=1+\frac{2 J_{0}^{\prime}(\sqrt{-i n \beta})}{\sqrt{-i n \beta J_{0}(\sqrt{-i n \beta})}}
$$

By means of equation (32) we may express the alternating current inductance and resistance of the circuit in terms of ber and bei functions of $\sqrt{n \beta}$, for since

it follows that

$$
J_{0}(\sqrt{-i n \beta})=\operatorname{ber} \sqrt{n \beta}+i \text { bei } \sqrt{n \beta}
$$

$\frac{2 J_{o}^{\prime}(\sqrt{-i n \beta})}{\sqrt{-i n \beta} J_{c}(\sqrt{-i n \beta)}}=\frac{2}{\sqrt{n \beta}} \frac{\text { ber' bei }- \text { ber bei }{ }^{\prime}+i\left(\text { ber ber }{ }^{\prime}+\text { bei bei' }\right)}{b e r^{2}+b e i^{2}}$ 
the argument of ber and bei being understood to be the real quantity $\sqrt{n \beta}$.

From equations (21), (32), and (34) it follows that

$$
\begin{gathered}
F_{1}(n \beta)=1-\frac{2 W(\sqrt{n \beta})}{\sqrt{n \beta} X(\sqrt{n \beta})} \\
F_{2}(n \beta)=\frac{2 Z(\sqrt{n \beta})}{\sqrt{n \beta} X(\sqrt{n \beta})}
\end{gathered}
$$

where

$$
\left\{\begin{array}{l}
W(x) \equiv \text { ber } x \text { bei }^{\prime} x \text {-bei } x \text { ber }^{\prime} x \\
Z(x) \equiv \text { ber } x \text { ber }^{\prime} x+\text { bei } x \text { bei }^{\prime} x \\
X(x) \equiv(\text { ber } x)^{2}+(\text { bei } x)^{2}
\end{array}\right.
$$

The functions $W$ and $Z$ occur with the same argument $\sqrt{n \beta}$ in the well-known expressions for the skin effect or alternating current inductance and resistance of a wire of radius $a$ in which the current flows parallel to the axis. ${ }^{4}$ However, the formula for the latter contain the ratios $\frac{W}{Y}$ and $\frac{Z}{Y}$ where $Y$ (in distinction from the $X$ here defined) is

$$
Y=\left(\operatorname{ber}^{\prime} x\right)^{2}+\left(\operatorname{bei}^{\prime} x\right)^{2}
$$

By making use of a table of ber and bei functions and their derivatives, and the equations (35), (36), and (37), one may compute the alternating-current inductance and resistance of the circuit without the necessity of computing the infinite series in equations (22) and (23). However, this series converges very rapidly for all values of $n \beta$, and it may be doubted whether the use of ber and bei functions would really result in a simplification of the computation. In order to find asymptotic or high frequency expressions for $L^{\prime}$ and $R^{\prime}$ it is more convenient to resort to the corresponding expansions for Bessel's functions.

When $n \beta$ is large and positive the asymptotic expansion of the Bessel's function gives

$$
F_{1}(n \beta)+i F_{2}(n \beta) \equiv-\frac{J_{2}(\sqrt{-i n \beta})}{J_{0}(\sqrt{-i n \beta})} \approx 1-\sqrt{\frac{2}{n \beta}}+i \sqrt{\frac{2}{n \beta}}+\cdots
$$

This gives the following asymptotic expansions for the apparent inductance and resistance of the circuit for high frequency:

$$
\begin{gathered}
F_{1}(n \beta) \approx 1-\sqrt{\frac{2}{n \beta}} \\
F_{2}(n \beta) \approx \sqrt{\frac{2}{n \beta}} \quad \text { if } \frac{1}{\sqrt{n \beta}} \text { is small compared to } 1
\end{gathered}
$$

'B. S. Sci. Paper No. 169, p. 174. 
By means of equations (32) and (21) we may now express the periodic charge $Q_{\mathrm{n}}(t)$ corresponding to a periodic electromotive force of type (20) in the form ${ }^{5}$

where

$$
Q(t)=\frac{B_{\mathrm{n}} e^{\mathrm{in} t}}{L(i n)^{2}+\operatorname{Rin}+\frac{1}{C}+(1-\epsilon) L(i n)^{2} \frac{J_{2}(\sqrt{-i n \beta})}{J_{0}(\sqrt{-i n \beta})}}=\frac{B_{\mathrm{n}} e^{\ln t}}{f(\sqrt{-i n})}
$$

$$
f(\bar{z}) \equiv L z^{4}-R z^{2}+\frac{1}{C}+(1-\epsilon) L z^{4} \frac{J_{2}(z \sqrt{\beta})}{J_{0}(z \sqrt{\beta})}
$$

The functions $F_{1}$ and $F_{2}$ of equations (22) and (23) have been computed and plotted in Figures 2 and 3 against $x=\sqrt{n \beta}$. In Figure 4 are shown the observed and computed values of the resistance change with frequency for a certain coil whose core consisted of a large number of iron wires about $1 \mathrm{~mm}$ in diameter. The coil was not very long compared to its diameter, so that the demagnetizing effects of its ends probably account for most of the deviation between the observed and computed values. The observations were taken about two years ago by Dr. F. B. Silsbee, of this bureau. The curve was constructed by assuming that $\beta=4 \pi \mu \lambda a^{2}=10^{-4}$.

\section{CASE OF AN ARBITRARY APPLIED ELECTROMOTIVE FORCE}

When $V(t)$ is an arbitrary function of the time which like the current becomes identically zero at infinitely remote past and future time, it may be represented by the Fourier integral identity

$$
V(t)=\frac{1}{2 \pi} \int_{-\infty}^{\infty} d n e^{\operatorname{lnt}} \int_{-\infty}^{\infty} e^{-\ln t} V\left(t^{\prime}\right) d t^{\prime}
$$

The solution of equation (19) then is, by equation (42)

where

$$
\begin{aligned}
Q(t) & =\frac{1}{2 \pi} \int_{-\infty}^{\infty} \frac{d n e^{\mathrm{int}}}{f(\sqrt{-i n})} \int_{-\infty}^{\infty} e^{-\mathrm{lnt} t^{\prime}} V\left(t^{\prime}\right) d t^{\prime} \\
& =\int_{-\infty}^{t} V\left(t^{\prime}\right) \Omega\left(t-t^{\prime}\right) d t^{\prime}
\end{aligned}
$$

$$
\Omega(t)=\frac{1}{2 \pi} \int_{-\infty}^{\infty} \frac{e^{\mathrm{int}} d n}{f(\sqrt{-i n)}}
$$

S The periodic solution equation (42) may also be derived by assuming that $V(t), I(t)$, and $H(r, t)$ are each proportional to $e^{\text {int. }}$. In satisfying the partial difforential equation (1) with the boundary condition equation (2), the Bessel's function naturally makes its appearance in this form; that is, with argument $\sqrt{-i n \beta}$. The circuit equations corresponding to equations (13) to (19), inclusive, would then have to be applied; the final equation corresponding to equation (19) then becomes identical (for the periodic case) with equation (42). 


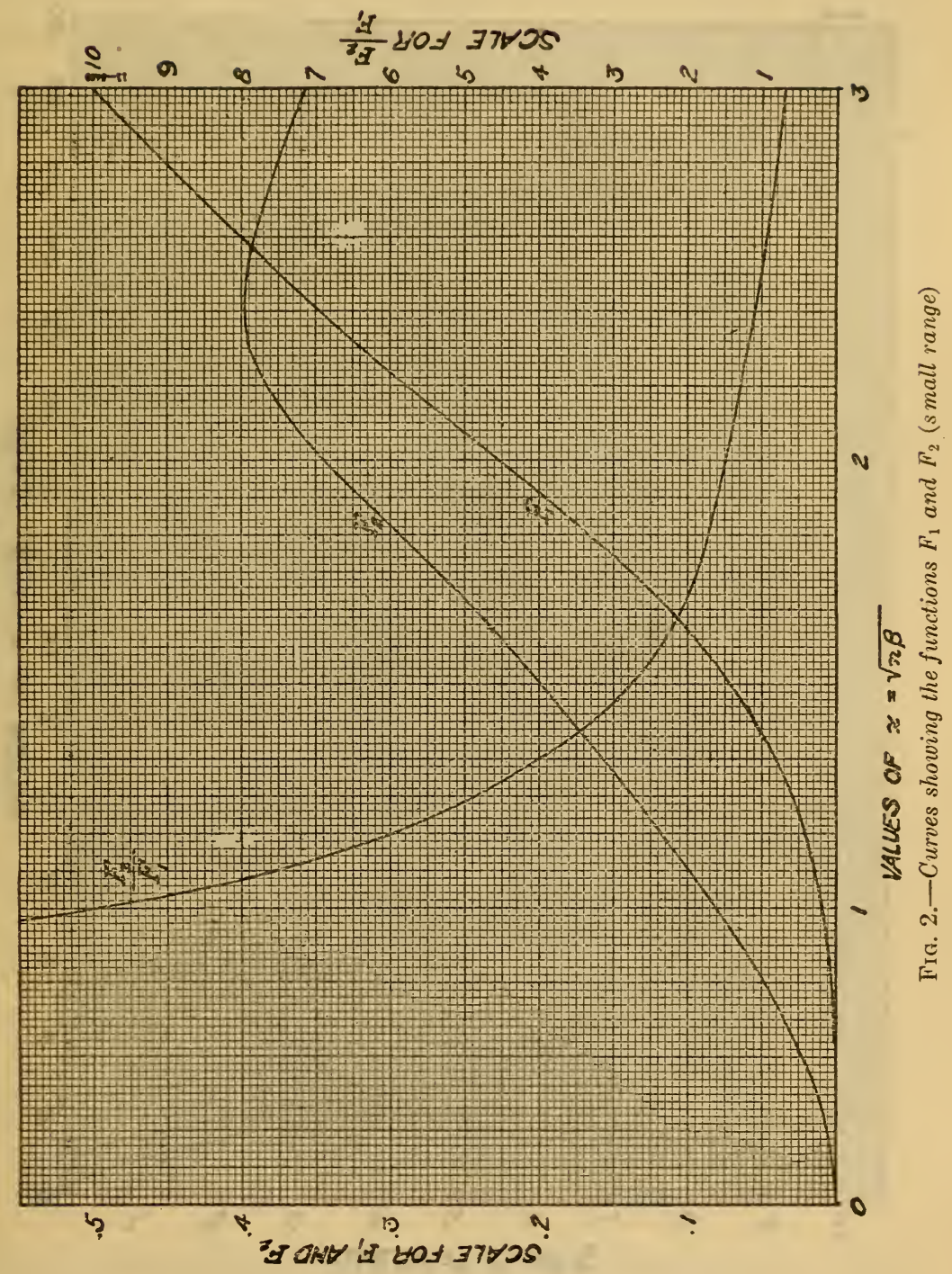




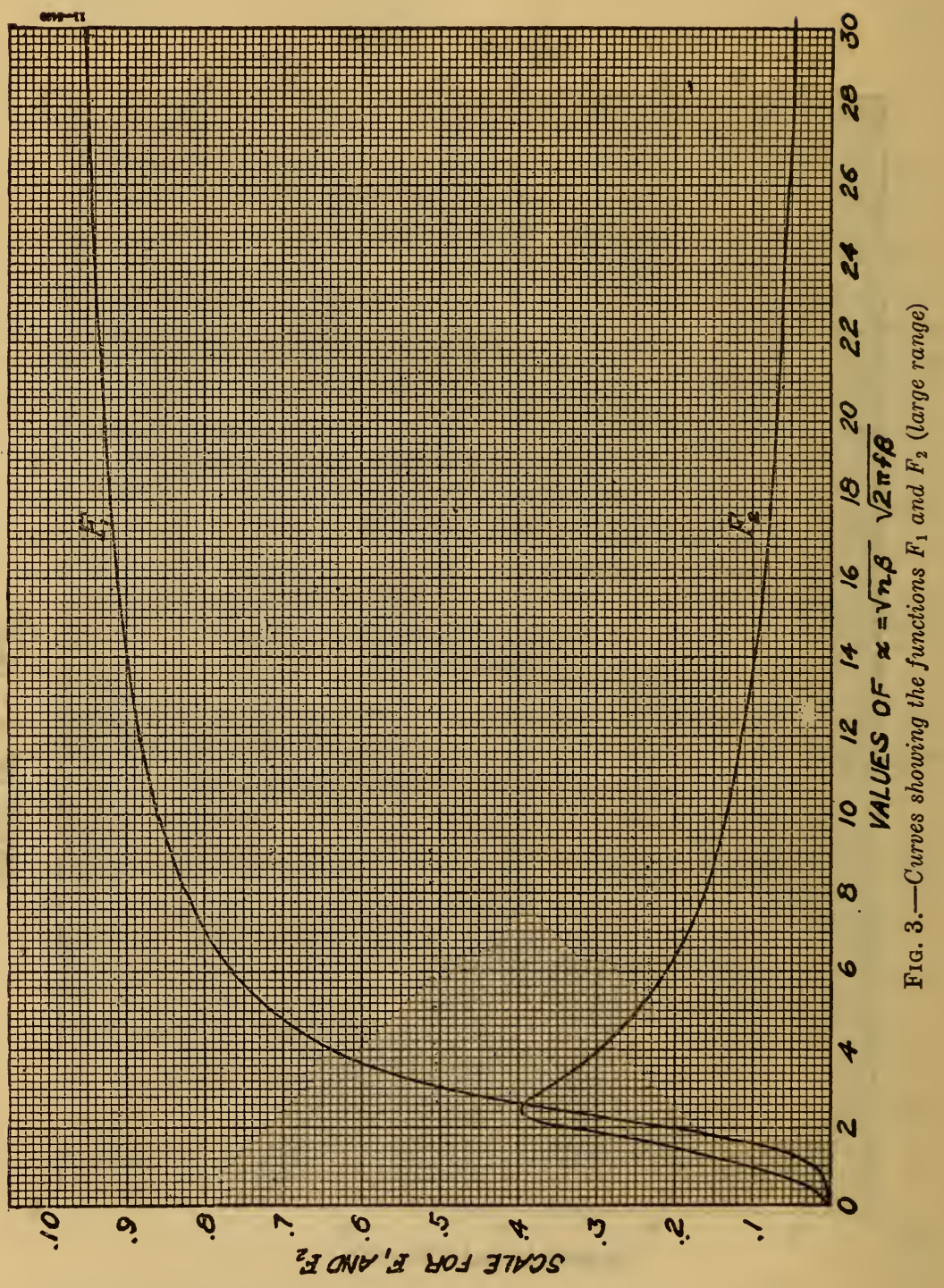


The justification for making the upper limit of $t^{\prime}$ in equation (45) equal to $t$ rather than $+\infty$ lies in the fact that $\Omega(t)$ is identically zero if $t$ is negative or zero (and hence $\Omega\left(t-t^{\prime}\right)$ is zero when $t^{\prime} \geqq t$ ). This depends upon the fact that (since $L$ is never zero and $\frac{1}{C}$ is not considered to be zero at present) the values of $n$ which are roots of $f(\sqrt{-i n})=0$ all lie in the upper half of the complex $n$ plane. Hence

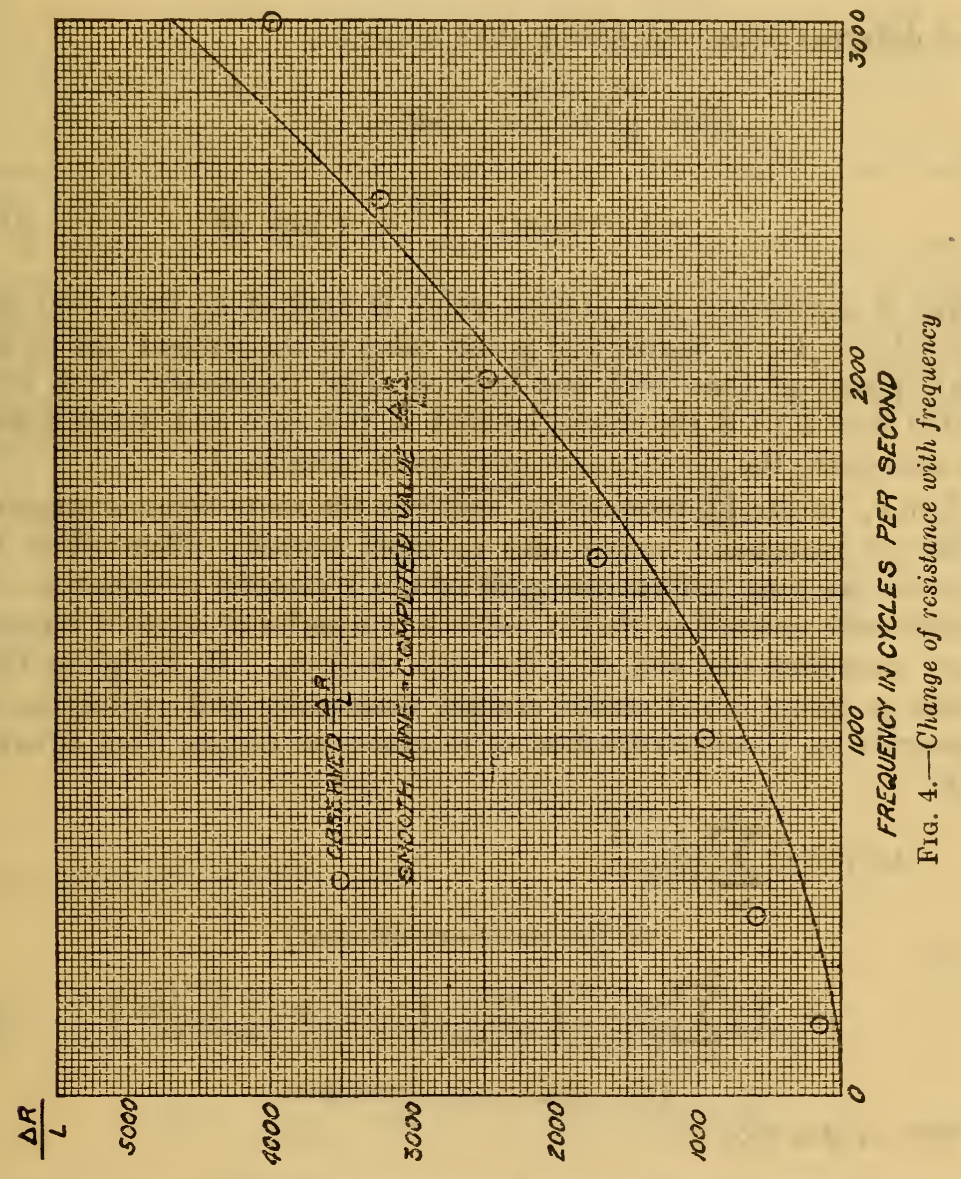

by deforming the path indefinitely downward in the complex $n$ plane, we find that $\Omega(t)$ vanishes when $t$ is negative or zero. It will be shown presently from the definition of the function $f$ that its roots are so situated, and this must be evident from general considerations since the system is dissipative. If it were not so we should obtain the absurd result that by the same downward displacement of the path a value of $Q(t)$ (or $I(t))$ at time $t$ of the type $A e^{(b+i w)\left(t-t^{\prime}\right)}$ due to a unit 
emf which is destined to be applied at time $t^{\prime}$ in the future-the more remote in the future the greater its effect now.

The physical interpretation of $\Omega(t)$ may be obtained from equation (45) by considering the case of an emf applied at time $t=0$ so that $\mathrm{V}(t)=0$ if $t<0$. Then equation (45) gives

$$
Q(t)=\int_{0}^{t} V\left(t^{\prime}\right) \Omega\left(t-t^{\prime}\right) d t^{\prime}=\int_{0}^{t} V\left(t-t^{\prime}\right) \Omega\left(t^{\prime}\right) d t^{\prime}
$$

and differentiating and noting that $\Omega(0)=0$

$$
\begin{aligned}
I(t) & =\int_{0}^{t} V\left(t^{\prime}\right) \dot{\Omega}\left(t-t^{\prime}\right) d t^{\prime} \\
& =V(+0) \Omega(t)+\int_{0}^{t} \dot{V}\left(t-t^{\prime}\right) \Omega\left(t^{\prime}\right) d t^{\prime}
\end{aligned}
$$

Thus if a constant emf of unit value is applied at time $t=0$ then $I(t)=\Omega(t)$. Or in words, $\Omega(t)$ is the value of the current due to unit emf applied at time $t=0$, and held constant thereafter. It is to be noted that $\Omega(0)$ is the initial current in this case and it will always be zero since the circuit must have an inductance $L$.

Let $n_{\mathrm{k}}$ be the $k^{\text {th }}$ root of $f(\sqrt{-i n})=0$, the roots being arranged in order of increasing magnitudes of their moduli. Then when $t$ is positive we may deform the path of the $n$ integral in equation (46) indefinitely upward so that it reduces to a series of contour integrals each encircling one and only one pole at $n=n_{\mathrm{k}}$. By shrinking these loops to infinitesimal circles around these poles and evaluating the integrals by Cauchy's method of residues, we obtain from equation (46)

$$
\begin{aligned}
& \Omega(t)=-2 \sum_{k=1}^{\infty} \frac{z_{\mathrm{k}} e^{-z_{\mathrm{k}}{ }^{2} t}}{f^{\prime}\left(z_{\mathrm{k}}\right)} \\
& \text { (if the roots are distinct) } \\
& =-\left(2 \sum_{k=3}^{\infty} \frac{z_{\mathrm{k}} e^{-z_{\mathrm{k}}{ }^{2} \mathrm{t}}}{f^{\prime}\left(z_{\mathrm{k}}\right)}\right)-\frac{4 e^{-z_{1} \mathrm{t}^{\mathrm{t}}}}{f^{\prime \prime}\left(z_{1}\right)}\left(1-2 z_{1}^{2} t-\frac{z_{1}}{3} \frac{f^{\prime \prime \prime}\left(z_{1}\right)}{f^{\prime \prime}\left(z_{1}\right)}\right)
\end{aligned}
$$

where $z_{\mathrm{k}}$ is a root of

$$
\text { (if } z_{1} \text { and } z_{2} \text { are coincident) }
$$

$$
f(z) \equiv L z^{4}-R z^{2}+\frac{1}{C}+(1-\epsilon) L z^{4} \frac{J_{2}(z \sqrt{\beta})}{J_{0}(z \sqrt{\beta})}=0
$$

If $z_{\mathbf{k}}=x_{\mathbf{k}}+i y_{\mathbf{k}}$ then $\left|y_{\mathrm{k}}\right|<x_{\mathbf{k}}>0$. If we differentiate this expression for $f(z)$ with respect to $z$ and make use of the properties of Bessel's functions and also of the fact that $z_{\mathrm{k}}$ is a root of $f(z)=0$, then the equation (47) reduces to the following when the roots are distinct: 


$$
\Omega(t)=\sum_{k=1}^{\infty} \frac{e^{-{ }^{z_{\mathbf{k}}{ }^{2 t}}}}{-L z_{\mathbf{k}}{ }^{2}+\frac{1}{C z_{\mathbf{k}}{ }^{2}}-\frac{\beta L}{4} g(z)}
$$

where

$$
g(z)=\left[(1-\epsilon) z_{\mathrm{k}}{ }^{4}-2\left(z_{\mathrm{k}}{ }^{4}-\frac{R{z_{\mathrm{k}}}^{2}}{L}+\frac{1}{L C}\right)+4 \frac{\left(z_{\mathbf{k}}{ }^{4}-\frac{R z_{\mathrm{k}}{ }^{2}}{L}+\frac{1}{L C}\right)^{2}}{(1-\epsilon) z_{\mathbf{k}}{ }^{4}}\right]
$$

This requires obvious modification in case the first two roots are coincident. However, if the roots can be found, on the assumption that they are distinct the expression for $\Omega(t)$ may then be evaluated in case these two roots approach coincidence. When $\beta$ is small the denominator in equation (49) is arranged in the order of magnitude of the terms.

The completion of the problem calls for a discussion of the roots of $f(z)=0$ and an examination of methods of determining them. Before considering this in general we may first confirm the truth of equation (49) in case the conductivity of the core wires is negligibly small so that there are no eddy currents and $\beta$ is zero. The characteristic equation (48) then reduces to

$$
L z^{4}-R z^{2}+\frac{1}{C}=0 \text { so that }\left\{\begin{array}{l}
z_{1}^{2}=b_{0}+i w_{0} \\
z_{2}{ }^{2}=b_{0}-i w_{0}
\end{array}\right\} \begin{aligned}
& b_{0}=\frac{R}{2 L} \\
& w_{0}=\sqrt{\frac{1}{L C}-\frac{R^{2}}{4 L^{2}}}
\end{aligned}
$$

Hence

$$
\begin{aligned}
& -L_{1} z_{1}^{2}+\frac{1}{C z_{1}^{2}}=-2 i L w_{0} \\
& -L z_{2}^{2}+\frac{1}{C z_{2}^{2}}=2 i L w_{0}
\end{aligned}
$$

and this gives in equation (49)

$$
\Omega(t)=\frac{1}{L} \frac{e^{-\mathrm{b}_{\mathrm{o}} \mathrm{s}} \sin w_{\mathrm{o}} t}{w_{\mathrm{o}}}
$$

This used in equation (45) gives the well-known solution

$$
\begin{aligned}
Q(t) & =\frac{1}{w_{0} L} \int_{0}^{\infty} V\left(t^{\prime}\right) e^{-\mathrm{b}\left(t-t^{\prime}\right)} \sin w_{0}\left(t-t^{\prime}\right) d t^{\prime} \\
& =C V_{0}\left\{1-e^{-b_{0} t}\left(\cos w_{0} t+b \frac{\sin }{w_{0}} w_{0} t\right)\right\}
\end{aligned}
$$

if $V$ is constant. Differentiating this gives

$$
I(t)=\frac{V_{\mathrm{o}}}{L} e^{-\mathrm{b}_{\mathrm{o}} t} \frac{\sin w_{\mathrm{o}} t}{w_{\mathrm{o}}}=V_{\mathrm{o}} \Omega(t)
$$

(See equation $\left(45^{\prime \prime}\right)$.) 
It is to be noted that equations (51), (52), and (53) preserve their meaning even if $w_{\mathrm{o}}$ is zero or imaginary, which illustrates the statement made above about two roots becoming identical as do $z_{1}$ and $z_{2}$ when $w_{0}=0$. We may now examine the general case where $\beta$ is not equal to zero and the eddy currents, therefore, exert their influence upon the current. If we let

$$
z=\frac{x}{\sqrt{\beta}}
$$

the characteristic equation (48) becomes

$$
-\frac{1}{1-\epsilon}\left[1-\frac{\beta R}{L} \frac{1}{x^{2}}+\frac{\beta^{2}}{L C} \frac{1}{x^{4}}\right]=\frac{J_{2}(x)}{J_{0}(x)}=-1-\frac{2 J_{0}^{\prime}(x)}{x J_{0}(x)}
$$

Since the coefficients of equation (55) are all real, it follows that for each complex root there must be another corresponding complex root which is conjugate to it. We are only interested in those roots which are positive reals and those complex roots whose real parts are positive. This equation contains three real positive parameters, namely, $\frac{1}{1-\epsilon}, \frac{\beta R}{L}$ and $\frac{\beta^{2}}{L C}$. The pure ratio $\epsilon$ may in certain limiting cases be zero, but in general it must be less than 1 and greater than or equal to zero. The parameters $\frac{\beta R}{L}$ and $\frac{\beta^{2}}{L C}$ may have any positive value, or zero. When $\beta$ is not equal to zero, the equation (55) always has an infinite number of positive real roots (corresponding to time constants of the system). In addition to these, it has two "principal roots" which are real or complex, depending upon the values of the three parameters.

The real roots may be found as the abscissa of the points of intersection of the curves $y(x)$ and $Y(x)$, where

$$
\begin{gathered}
y(x) \equiv-\frac{1}{1-\epsilon}\left[1-\frac{\beta R}{L} \cdot \frac{1}{x^{2}}+\frac{\beta^{2}}{L C} \cdot \frac{1}{x^{4}}\right] \\
Y(x) \equiv \frac{J_{2}(x)}{J_{\mathrm{o}}(x)}=-1-\frac{2 J_{\mathrm{o}}^{\prime}(x)}{x J_{\mathrm{o}}(x)}=4 x^{2} \sum_{\mathrm{s}=1}^{\infty} \frac{1}{a_{\mathrm{s}}{ }^{2}\left(a_{\mathrm{s}}{ }^{2}-x^{2}\right)}
\end{gathered}
$$

The curve $Y(x)$ may be plotted once for all as shown in the Figure 5. Its infinities are the roots of $J_{0}(x)=0$, its zeros the roots of $J_{2}(x)$. If $C$ is finite, the curve $y(x)$ is $-\infty$ when $x=0$, and approaches 
$-\frac{1}{1-\epsilon}$ when $x=\infty$. It has no minimum, but one maximum value which is given by

$$
y_{\mathrm{m}}=\frac{L C}{1-\epsilon}\left[\left(\frac{R}{2 L}\right)^{2}-\frac{1}{L C}\right]
$$

which occurs at

$$
x_{\mathrm{m}}=\sqrt{\frac{2 \beta}{R C}}
$$

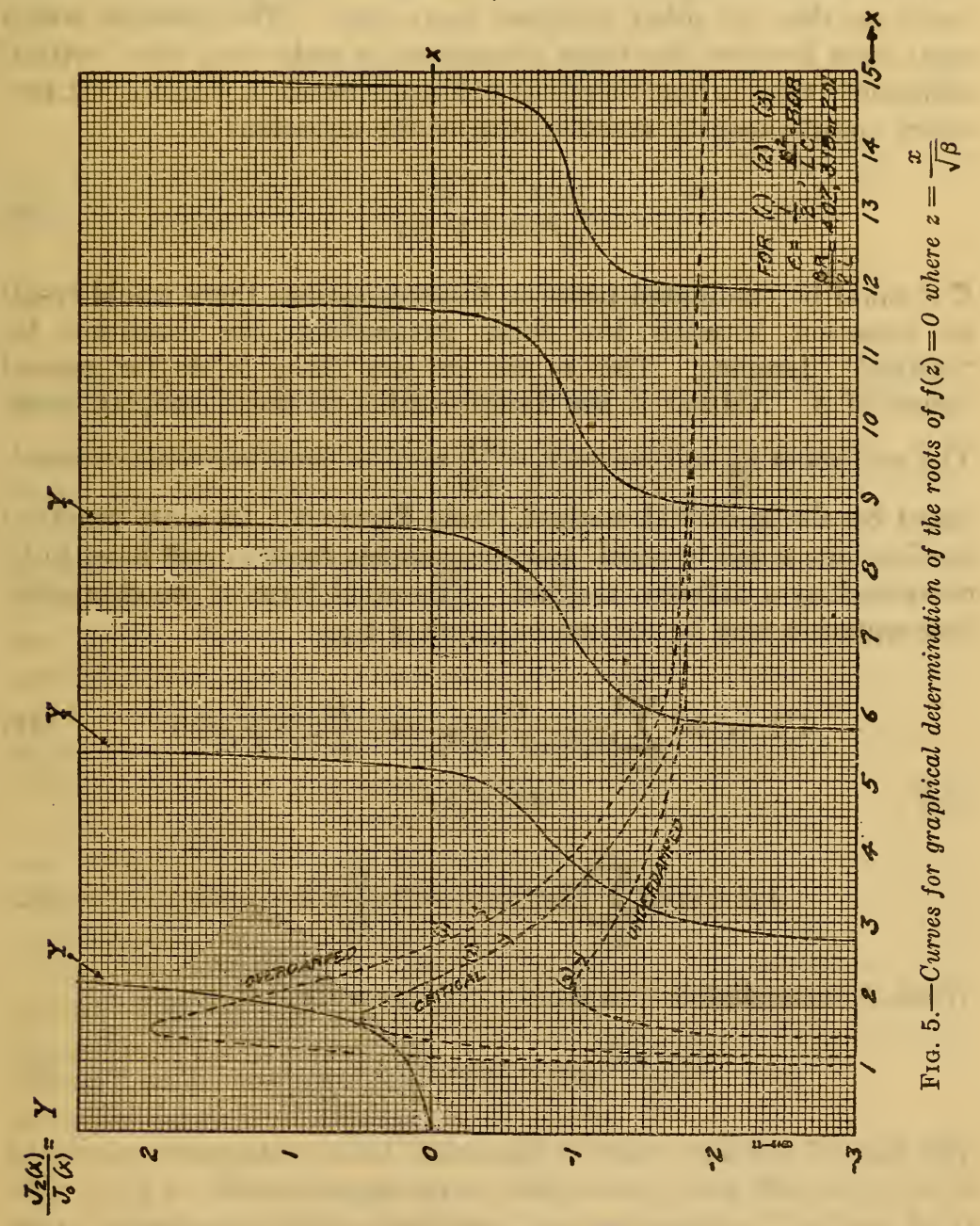

If the maximum value $y_{\mathrm{m}}$ is positive, the curve $y(x)$ crosses the axis twice. It is evident from the figure that the curve $y$ will always cut every branch of the $Y$ curve except possibly the first (principal branch). If $R$ is sufficiently large, it is evident that the $y$ curve will 
cut this principal branch in two distinct points if $C$ is finite (in one only if $C=\infty$; that is, no condenser). In this case all roots are real. If then we imagine that $R$ decreases, the other parameters being fixed, a critical value of $R$ will be reached (if $C$ is finite) for which the $y$ curve just touches the $Y$ curve. The two roots are identical. For smaller values of $R$ these two roots are conjugate complex roots and the system might be called "underdamped," but no further decrease in $R$ can make the $y$ curve become tangent to any branch of the $Y$ curve so that no other complex roots exist. The relation which must exist between the three parameters in order that this "critical damping" exist is that there is a value of $x$ between 0 and $a_{1}=2.405$, which simultaneously satisfies both of the equations

$$
\left\{\begin{array}{l}
y(x)=Y(x) \\
y^{\prime}(x)=Y^{\prime}(x)
\end{array}\right.
$$

If $x$ could be eliminated between these equations there would result an equation between the three parameters-the condition for "critical" damping. This is not an easy thing to do for general values of $\beta$. Neither is the determination of these complex roots. The real roots $x_{\mathrm{k}}$ and hence $z_{\mathrm{k}}=\frac{x_{\mathrm{k}}}{\sqrt{\beta}}$ may be most conveniently evaluated by the graphical method, using Figure 5. In most practical applications $\beta$ will be small and the complex roots $z_{\mathrm{k}}$ will have to be computed by a different method. The exact form of the characteristic equation may be written in terms of $z$ as

$$
4(1-\epsilon) \beta z^{6} \sum_{s=1}^{\infty} \frac{1}{a_{s}^{2}\left(a_{s}^{2}-\beta z^{2}\right)}+z^{4}-\frac{R}{L} z^{2}+\frac{1}{L C}=0
$$

or if

$$
\left|\beta z^{2}\right|<a_{1}^{2}
$$

$$
4(1-\epsilon) \beta z^{8} \sum_{\mathrm{n}=\mathrm{o}}^{\infty} \beta^{\mathrm{n}} z^{2 \mathrm{n}} S_{2 \mathrm{n}+4}+z^{4}-\frac{R}{L} z^{2}+\frac{1}{L C}=0
$$

When $\beta z^{2}$ is negligible compared to 1 , this reduces to

$$
\frac{(1-\epsilon) \beta}{8}\left(z^{2}\right)^{3}+\left(z^{2}\right)^{2}-\frac{R}{L} z^{2}+\frac{1}{L C}=0
$$

The pair of complex roots of this cubic for $z^{2}$ (where $z^{2}=a \pm i b$ and $a>0, b>0$ ) will give the complex roots approximately of $f(z)$. The third root is $z^{2}=\mathrm{a}$ negative real, and this is quite inapplicable to the problem, as we have shown there are no such roots of $f(z)$. This third root in fact violates the assumption that $\beta z^{2}$ is small. The other roots (real) must be determined graphically by the use of Figure 5. 
By means of equation (57) we may obtain the relation which must exist between the parameters for "critical" damping when $\beta z$ is small. Placing $z^{2}=\xi$, the condition is that the equation

$$
\frac{(1-\epsilon) \beta}{8} \xi^{3}+\xi^{2}-\frac{R}{L} \xi+\frac{1}{L C}=0
$$

should have a double root which is a positive real, and $\xi$ must, therefore, be a root also of

$$
\frac{3(1-\epsilon) \beta}{8} \xi^{2}+2 \xi-\frac{R}{L}=0
$$

Solving equation (65) for $\xi$ and placing this value in equation (64) gives the condition for critical damping (when $\frac{\beta R}{2 L}$ is small).

$$
\frac{1}{L C}=\left(\frac{R}{2 L}\right)^{2}\left[1-\frac{(1-\epsilon) \beta}{8}\left(\frac{R}{2 L}\right)\right]
$$

Since $\frac{\beta R}{2 L}$ must be small for this relation to be valid, the bracket is 1 minus a small correction due to the existence of eddy currents. This shows that for a given inductance and capacity the circuit will be critically damped with a larger resistance than if there were no eddy currents. This might seem paradoxical if one thought of the effect of the eddy currents as equivalent to a mere increase in resistance of the circuit. But it must be remembered that they also affect its equivalent inductance. Moreover, the phrase "critical damping" as here used is not intended to imply that the system behaves like a single circuit or a finite number of circuits, since there are always an infinite number of real time constants of the system. The words "critical damping," "underdamped," and "overdamped" are here used with reference to the two principal roots, as in the three dotted curves of Figure 5. In this sense it is evident that if the circuit were underdamped in the absence of eddy currents, then it would always be underdamped with them, and in certain cases if overdamped without them, then their presence would render it underdamped. This corresponds to the case where the $y$ curve in Figure 5 might cross the axis of $x$ without rising high enough to cut the principal branch of $Y$ curve. Whatever the nature of the two principal roots, it is evident that for large values of $\beta$ the real exponential terms of $\Omega(t)$ corresponding to the remaining (real) roots will exert an important influence upon the course of the current. 
If we allow the capacity $C$ of the condenser to increase indefinitely, the first root of $f(z)=0$ approaches zero in such a manner that its principal part is given by $\frac{1}{C z_{1}{ }^{2}}=R$ so that the contribution of the first root (for $k=1$ ) to the second member of equation (49) is just $\frac{1}{R}$.

The second root $z_{2}{ }^{2}=\frac{x_{2}{ }^{2}}{\beta}$ is found from the abscissa $x_{2}$ of the points of intersection of the curve

$$
y(x)=-\frac{1}{1-\epsilon}\left(1-\frac{\beta R}{L} \cdot \frac{1}{x^{2}}\right)
$$

with the curve $Y(x)=\frac{J_{2}(x)}{J_{0}(x)}$ and if $\beta$ is also small (as it is in all practical cases) this value of $x_{2}^{2}$ is the small positive root of

which is

$$
\frac{x_{2}{ }^{6}}{24}+x_{2}{ }^{4}+\frac{8}{1-\epsilon} x_{2}{ }^{2}-\frac{8 \beta R}{(1-\epsilon) L}=0
$$

$$
\frac{x_{2}^{2}}{\beta}=z_{2}^{2}=\frac{R}{L}\left[1-\frac{(1-\epsilon) \beta R}{8 L}\right]
$$

The remaining roots are the positive abscissa of the points of intersection of the curves of Figure $5 Y(x)=\frac{J_{2}(x)}{J_{0}(x)}$ and the curve $y=-\frac{1}{1-\epsilon}\left[1-\frac{\beta R}{L x^{2}}\right]$. If $\beta$ is so small that $y\left(a_{1}\right)$ is very close to its asymptotic value $-\frac{1}{1-\epsilon}$ and if this is a large negative value, the roots are very approximately all the roots of $J_{0}(x)=0$ beginning with the first. On the other hand, if $\epsilon$ is zero the roots are the roots of $J_{1}(x)=0$. In general they will be between these two extremes when $\beta$ is very small. Thus if $\epsilon$ is so large and $\beta$ so small that the $y$ curve has passed its maximum and reached a negative value as low as -5 by the time $x$ reaches the value $a_{1}=2.405$, then all the roots lie near the roots of $J_{0}$. In this case, if we let $x_{\mathrm{s}}=a_{\mathrm{s}}\left(1+\xi_{\mathrm{s}}\right)$ where $\xi_{\mathrm{s}}$ is small and $\alpha_{\mathrm{s}}$ is the $s^{\text {th }}$ root of $J_{0}(x)=0$, then it is readily found that when $\xi_{s}$ is small it is given by

$$
\xi_{\mathrm{s}}=\frac{2}{1-2 a_{\mathrm{s}}\left[1+y\left(a_{\mathrm{s}}\right)\right]}
$$

In general, the graphical method, using Figure 5, will be most convenient for finding the remaining roots. In conclusion it may be interesting to point out an analogy.

When the magnetizing current $I(t)$ is periodic of frequency $\frac{n}{2 \pi}$ so that $I(i)$ is the real part of $I_{\mathrm{n}} e^{\mathrm{Int}}$ and the magnetizing force is the real 
part of $4 \pi N I_{\mathrm{n}} e^{\operatorname{lnt}}$ then the field $H$ in a core wire is given by equation (4) as

$$
H(r, t)=4 \pi N I_{\mathrm{n}} e^{\operatorname{lnt} \mathrm{t}} \frac{J_{0}\left(\frac{r}{a} \sqrt{-i n \beta}\right)}{J_{\mathrm{o}}(\sqrt{-i n \beta})} \text { (real part) }
$$

This follows immediately from the fact that within the core wire $H$ satisfies the equation

$$
\frac{\beta}{a^{2}} \dot{H}=\frac{\partial^{2} H}{\partial r^{2}}+\frac{1}{r} \frac{\partial H}{\partial r}
$$

and $H$ reduces to $4 \pi N I_{\mathrm{n}} e^{\text {Int }}$ (real part) when $r=a$. The problem analogous to this is that where an electric field, the real part of $E_{\mathrm{n}} e^{\operatorname{lnt}}$, is applied to a cylindrical wire of radius $a$. In this case the electric field $E$ which is parallel to $z$ satisfies the equation

$$
\frac{\beta}{a^{2}} \dot{E}=\frac{\partial^{2} E}{\partial r^{2}}+\frac{1}{r} \frac{\partial E}{\partial r} \text { and } E=E_{\mathrm{n}} e^{\operatorname{lnt}} \text { when } r=a
$$

The analogy between $H$ and $E$ in the two cases is perfect, and $E(r, t)$ is given by

$$
E(r, t)=E_{\mathrm{n}} e^{\operatorname{lnt}} \frac{J_{\mathrm{o}}\left(\frac{r}{a} \sqrt{-i n \beta}\right)}{J_{\mathrm{o}}(\sqrt{-i n \beta})} \text { (real part) }
$$

This is the equation for the "skin effect" in the cylinder with steady alternating current. Similarly, the distribution of current when the applied electric field is an arbitrary function of the time $E(t)$ is given by the equation analogous to equation (12)

$$
E(r, t)=E(t)-\int_{-\infty}^{t} \dot{E}(\tau) d \tau \sum_{s=1}^{\infty} \frac{2 J_{0}\left(\frac{r}{a} a_{s}\right)}{a_{s} J_{1}\left(a_{s}\right)} e^{-\alpha_{s}{ }^{2}}(t-\tau)
$$

It is not improbable that this equation for the transient current may be found in the literature. 


\section{SUMMARY}

It has been shown in Section II that if a current $I(t)$ flows in a circuit which consists of uniform winding around a cylindrical core of $N^{\prime}$ equal parallel wires, each of circular section of radius $a$, conductivity $\lambda$, and magnetic permeability $\mu$, and if the circuit has a total resistance $R$, a series capacity $C$, and an inductance $L$, all referred to zero frequency, then the course of the current under the influence of an arbitrary, applied electromotive force $V(t)$, together with the influence of the eddy currents in the core wire, will be determined by the equation

$L \ddot{Q}(t)+R \dot{Q}(t)+\frac{Q(t)}{C}(1-\epsilon) L \beta \int_{0}^{\infty} \dddot{Q}(t-\beta \tau) d \tau \sum_{\mathrm{s}=1}^{\infty} \frac{4}{a_{\mathrm{B}}^{2}} e^{-\alpha_{\mathrm{e}}{ }^{2} \tau}=V(t)$

where $Q(t)$ is the charge on the condenser, so that $I(t)=\dot{Q}(t)=\frac{d Q(t)}{d t}$ and $\beta$ is a constant depending upon the core wires, and defined by

$$
\beta=4 \pi \mu \lambda a^{2}
$$

The numerical constant $a_{8}$ is the $s^{\text {th }}$ positive root of $J_{0}(a)=0$ and the pure ratio $\epsilon$ is a positive number, which is always less than 1 and defined by

$$
1-\epsilon=\frac{4 \pi N^{2} \mu S_{\mu}}{L}
$$

$N$ being the number of turns of the windings per $\mathrm{cm}$ length along the cylinder, $\mu$ the permeability of the core wire, and $S_{\mu}$ the total cross section of the core wires $=N^{\prime} \pi a^{2}$ where $N^{\prime}$ is their number.

In Section III it is shown that for the case of a periodic applied electromotive force of frequency $f=\frac{n}{2 \pi}$, the steady periodic current is the same as if the circuit possessed the inductance $L^{\prime}$ and the resistance $R^{\prime}$, both being functions of the frequency and given by

$$
\begin{gathered}
\frac{L^{\prime}-L}{L} \equiv \frac{\Delta L}{L}=-(1-\epsilon) F_{1}(n \beta)=-(1-\epsilon) \sum_{\mathrm{s}=1}^{\infty} \frac{4 n^{2} \beta^{2}}{a_{\mathrm{s}}{ }^{2}\left(a_{\mathrm{s}}{ }^{4}+n^{2} \beta^{2}\right)} \\
\frac{R^{\prime}-R}{n L} \equiv \frac{\Delta R}{n L}=(1-\epsilon) F_{2}(n \beta)=(1-\epsilon) \sum_{\mathrm{s}=1}^{\infty} \frac{4 n \beta}{a_{\mathrm{s}}{ }^{4}+n^{2} \beta^{2}}
\end{gathered}
$$

The functions $F_{1}$ and $F_{2}$ are expressed as power series in $n \beta$ by ercuations (26) and (27), which are only valid if $n \beta<a_{1}^{2}=(2.405)^{2}$. They are also expressed in terms of ber and bei functions and their derivatives, with argument $\sqrt{n b}$ in the equations (35) and (36). They 
are plotted to two scales in Figures 1 and 2. It is also shown that for high frequencies they take the asymptotic forms

$$
\begin{gathered}
F_{1}(n \beta) \approx 1-\sqrt{\frac{2}{n \beta}} \\
F_{2}(n \beta) \approx \sqrt{\frac{2}{n \beta}}
\end{gathered}
$$

In Section IV it is shown that under the influence of an arbitrary electromotive force $V(t)$, which is applied at time $t=-\infty$, the current will be given by

$$
I(t)=\int_{-\infty}^{t} V\left(t^{\prime}\right) \dot{\Omega}\left(t-t^{\prime}\right) d t^{\prime}
$$

where $\Omega(t)$ is the current that would be produced by a constant electromotive force applied at time $t=0$, if conditions had remained static previous to that time.

This characteristic function $\Omega(t)$ is studied in detail. It is expressed in the form

$$
\Omega_{\mathrm{k}=1}^{\infty}(t)=-2 \sum \frac{z_{\mathrm{k}} e^{-z_{\mathrm{k}}{ }^{2} t}}{f^{\prime}\left(z_{\mathbf{k}}\right)}
$$

if the roots are distinct

$$
=-2 \sum_{k=3}^{\infty} \frac{z_{\mathrm{k}} e^{-z_{\mathrm{k}}{ }^{2} \mathrm{t}}}{f^{\prime}\left(z_{\mathrm{k}}\right)}-\frac{-4 e^{-z_{1} 2 \mathrm{t}}}{f^{\prime \prime}\left(z_{1}\right)}\left(1-2 z_{1}^{2} t-\frac{z_{1} f^{\prime \prime \prime}\left(z_{1}\right)}{3 f^{\prime \prime}\left(z_{1}\right)}\right)
$$

if $z_{1}$ and $z_{2}$ are coincident, where $z_{\mathrm{k}}$ is a root of

$$
f(z)=0=L z^{4}-R z^{2}+\frac{1}{C}+(1-\epsilon) L z^{4} \frac{J_{2}(z \sqrt{\beta})}{J_{0}(z \sqrt{\beta})}
$$

The first two roots may be either real or complex, all the others real. The curve (fig. 5) is plotted to facilitate the determination of these roots in any numerical case.

Washington, May 6, 1926. 